\title{
Improved Content-Based Image Retrieval Technique for Query Generation in Mobile Networks
}

\author{
Barinder Kaur, Madan Lal, Jagroop Kaur
}

\begin{abstract}
Image database searching is in rapid growth with an advancement in multimedia technology. To manage these kinds of searches Content-Based Image Retrieval is an effective tool. In this paper, existing CBIR techniques are analyzed and a new technique has been proposed which works based on Region-Based Convolutional Neural Network (RCNN). In the proposed approach first of all image dataset is uploaded to cloud and features are stored in a storage. Then Query image is enhanced, uploaded and features are extracted. After this feature set is compared with dataset and matched images are extracted and ranked as the closest match. Using this proposed methodology, the accuracy and precision values are compared and validated and it is observed that the proposed methodology shows better results than the existing techniques.
\end{abstract}

Keywords: CBIR (Content-Based Image Retrieval), CNN (Convolutional Neural Network), Image, GLCM (Grey-Level Co-Occurrence Matrix)

\section{INTRODUCTION}

Content-Based Image Retrieval is a tool by which images can be extracted from the image database using the features provided by query image. Content-based image retrieval is opposed to traditional concept-based approaches. In the Content based image retrieval content of images are analyzed rather than the information of the image such as keywords, metadata or tags. As the traditional techniques for image retrieval by entering the keywords and tags in large databases is a time-consuming task also it cannot be always accurate. In the modern techniques of content-based image retrieval the feature set of the images are extracted and the images are withdrawn according to indexing of the databases. This is the typical CBIR technique in which images closest to the features of image under examination is matched and results are obtained. [10], [11], [12]

\section{RCNN}

Region-Based Convolutional Neural Network (RCNN) is the classification scheme that can reduce the problem of selection

Revised Manuscript Received on August 27, 2020.

* Correspondence Author

Barinder Kaur*, Department of Computer Science and Engineering, Punjabi University, Patiala, India. E-mail: barindersaini007@gmail.com

Madan Lal, Department of Computer Science and Engineering, Punjabi University, Patiala, India. E-mail: mlgtb@rediffmail.com

Jagroop Kaur, Department of Computer Science and Engineering, Punjabi University, Patiala, India. E-mail: jagroop_80rediffmail.com

(C) The Authors. Published by Blue Eyes Intelligence Engineering and Sciences Publication (BEIESP). This is an open access article under the CC BY-NC-ND license (http://creativecommons.org/licenses/by-nc-nd/4.0/) of large number of regions. In this classification algorithm, selective search is used in which 2000 regions from images are extracted and is called region proposals. In this algorithm, firstly the region proposals are obtained then the edge boxes are constructed and those regions are trimmed from the image. Later Convolutional Neural Network (CNN) is to be applied and then SVM is used to refine the region proposal bounding boxes that will in turn trains the features of CNN.
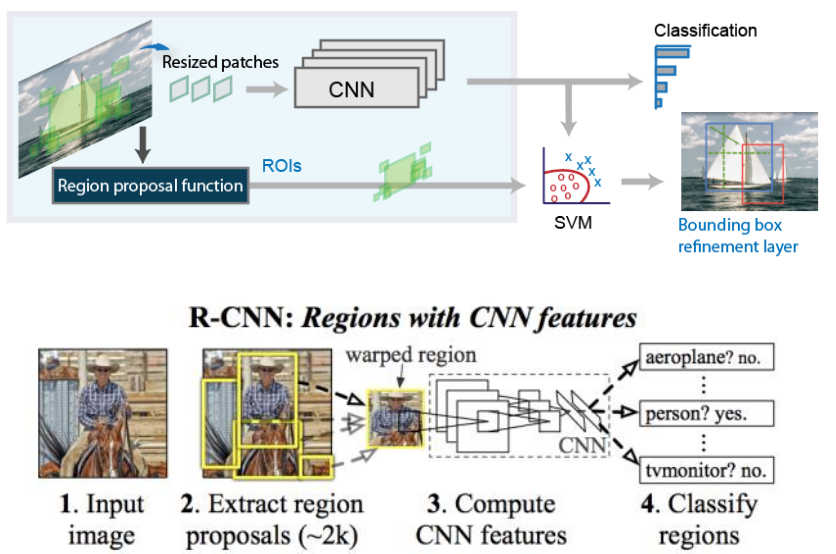

Fig. 1. RCNN

\section{RELATED WORK}

In this section, we have a tendency to summarize and discuss connected authentication ways employed in follow or projected within the literature to boost positive identification and authentication on the net and gift their limits.

Boparai, NK.; et al. [1] presents a hybrid scheme that consists of Neural Network and Genetic Algorithm. This hybrid structure decreases the semantic asymmetry between human observation and prediction done by machine by employing low-level component descriptors like HSV shading histograms, shading minutes and changes in wavelet that matches human perception. Execution result represents improved accuracy and review for the purposed technique.

Bose, S.; et al. [2] proposed a content-based image retrieval to extricate, from enormous (image) databases, a predetermined number of images comparable in visual and semantic substance to a supposed inquiry image utilizing Relevance Feedback (RF) component. This research was undertaken to upgrade accuracy of retrieval by revising framework that depends on shading highlights while early segmentation provides consolidation of shape data of image. The proposed approach was effective in improving the retrieval exactness essentially

Published By:

Blue Eyes Intelligence Engineering and Sciences Publication

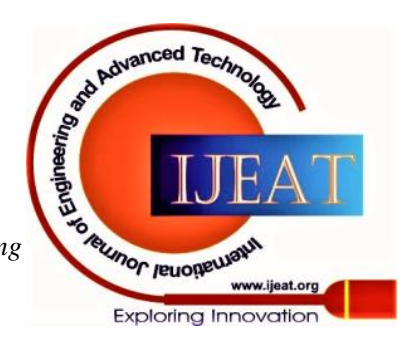




\section{Improved Content-Based Image Retrieval Technique for Query Generation in Mobile Networks}

Mistry, Y.; et al. [3] proposed a hybrid feature based on effective CBIR framework employing different separation measures. To upgrade accuracy, binarized factual image features (BSIF), Spatial features, shading and edge directivity descriptor features are utilized. Test results shows that the purposed approach performs better in accuracy in comparison to other existing frameworks.

Zhang, C.; et al. [4] proposed an algorithm that is based upon the DWT-DCT. First of all, medical image is encrypted and feature vector is separated and both are stored into encrypted medical image database in encrypted manner individually. Likeliness of two images is then calculated by standard relationship coefficient (NC). From result it can be concluded that proposed approach was better than other traditional once. Raza, A. et al. [5] proposed an image retrieval technique based upon associated essential visual texton histogram features (CPV-THF). It coordinates with visual and semantic data by generating a relation in various features of image. Shading surface along with power feature histogram are used as a key for image similarity and retrieval. The presentation of the proposed descriptor is contrasted and those of cutting-edge surface, shading, shape, and nearby example based CBIR descriptors.

Swati, ZNK.; et al. [6] proposed a CBIR technique that can detect brain tumor on T1-weighted differentiation upgraded attractive reverberation images (CE-MRI). In this a CNN based retrieval scheme is defined and apply closed structure metric to figuring out quantification of the likeliness of tumor in enquired image.

Cai, Y. et al. [7] proposed CBIR technique that is based upon CNN and Hash coding. In this structure Siamese network is used in which images are utilized as information source and a model is developed in which the similarity of the images is detected by weight and content of image. CNN plays an important role in each part of retrieval and similarity of image. On retrieval stage conservative double hash code of the query image is accomplished from the prepared network and is therefore contrasted and the hash codes of the database images.

Liu, F.; et al. [8] executed a genuine number calculation system and a separation and-vanquish CNN assessment convention to empower our structure to safely and proficiently assess the profound CNN with countless data sources and further proposed a protected image similitude scoring convention, which empowers the cloud servers to look at two images without knowing any data about their profound features. The far-reaching test results show that our system is productive and exact.

Table I: Comparative Analysis of existing CBIR techniques

\begin{tabular}{|c|c|c|c|c|}
\hline \multirow[b]{2}{*}{ Sr. No } & & \multirow[b]{2}{*}{ Methods } & \multirow[b]{2}{*}{$\begin{array}{c}\text { Research } \\
\text { Area }\end{array}$} & \multirow[b]{2}{*}{$\begin{array}{c}\text { Accura } \\
\text { cy }\end{array}$} \\
\hline & $\begin{array}{c}\text { Year of } \\
\text { Publica } \\
\text { tion }\end{array}$ & & & \\
\hline 1 & 2016 & CNN & $\begin{array}{c}\text { Real } \\
\text { number } \\
\text { computatio } \\
\text { n } \\
\text { mechanism }\end{array}$ & $96 \%$ \\
\hline 2 & 2017 & $\begin{array}{l}\text { SVM, } \\
\text { SURF }\end{array}$ & $\begin{array}{l}\text { Brain } \\
\text { Tumor }\end{array}$ & $94 \%$ \\
\hline 3 & 2017 & PCA & $\begin{array}{l}\text { Medical } \\
\text { Image }\end{array}$ & $94.5 \%$ \\
\hline
\end{tabular}

Retrieval

Image

Retrieval

space,

Wavelet

decomposi

tion

5

CNN and

Hash

Coding

2019 Deep

convolutio

nal neural

network

2019

$\begin{array}{cc}\text { Correlated } & \text { Image } \\ \text { primary } & \text { Retrieval } \\ \text { visual } & \\ \text { texton } & \\ \text { histogram } & \\ \text { features } & \end{array}$

Image

Retrieval

Image

Retrieval

$96 \%$

$96 \%$

\section{FEATURE EXTRACTION}

Feature extraction plays a significant role in CBIR system as it influences the retrieval rate of an image. It is one of the various boundaries dependent on which the features are chosen to extricate the images. The primary feature boundaries are portrayed as following.

Color: Color is one of the features of substance-based image retrieval frameworks to recover the image. Initial a shading space is utilized to speak to shading images. The RGB space where the dim level power is spoken to as the entirety of red, green and blue dark level forces. The Color Structure Descriptor (CSD) speaks to an image by both the nearby structure of the shading and the shading circulation of the image or image area.

Texture: Texture properties of images are defined structure and color properties of an image. These attributes are used in similarity matching and image retrieval. At each stage the CNN or RCNN or any other classification technique will be used so that the final results may be obtained.

Shape: Shape is an essential feature used to explain image content. Representation and interpretation of shape is difficult since 3-D objects are protruded onto 2-D plane. It represents the shape of the particular part of the image being worked on. Shapes are decided by applying segmentation on the selected image. Methods like Wilkinson 2008 and Tushabe use shape filters to find out the specific figures. It may require human involvement since segmentation is very hard to automate.

\section{METHODOLOGY}

The methodology represented below is about the flow chart. In flow chart all steps are mentioned that are used for e retrieval.

the proposed approach CBIR is used in cloud for mobile . In this mobile user can search data based upon quer (the help of CBIR. CBIR is a technique in which encrypted manner. 
After uploading of query image CBIR based proposed technique is used. In the proposed approach Region based CNN (RCNN) is used with which the layers of RCNN are fed with feature extracted from image and then these features are compared with dataset that is stored in cloud.

After comparison if feature is matched then images are extracted. Extracted images are sorted according to the \%age of features mapped.

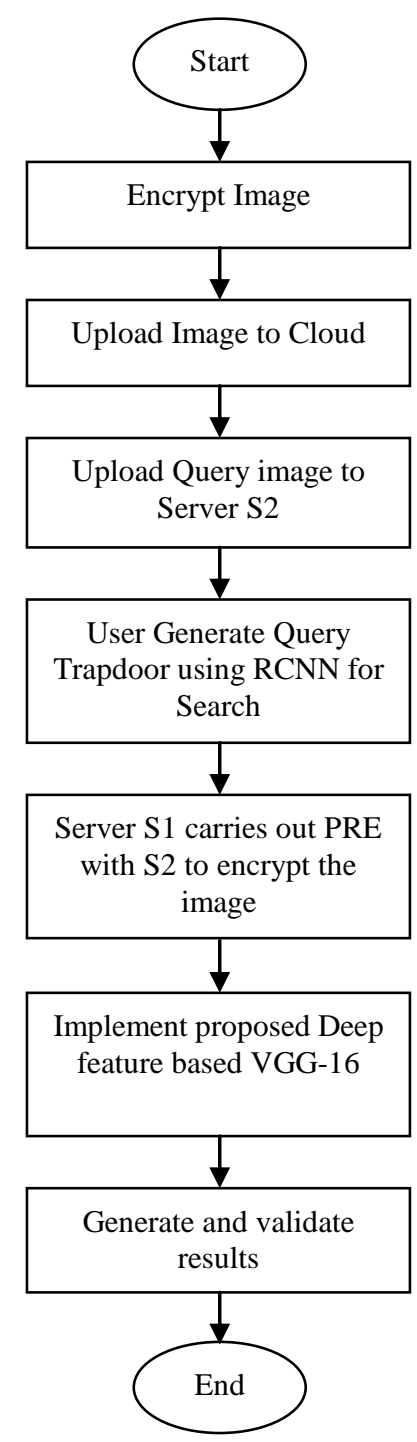

Fig. 2. Methodology Used

In the very first step, the pre-processing of image is done in which the image is filtered and converted gray scale into binary image with the help of otsu method.

Dv $=$ Dataset

$\mathrm{N}=$ Number of Images in dataset

$\mathrm{P}=$ Pixels of Image (Component of image)

Cen $=$ centroid point

$\mathrm{F}=$ Features

$\mathrm{Q}=$ Image for query

number of components and feature vectors of images are saved in database Dv.

Phase 1: Creation of Dv database:

Step 1: for $k=1: N * / N$ number of images; */read the $\mathrm{k}^{\text {th }}$ image from Dd

Step 2: for $\mathrm{I}=1: \mathrm{P}_{\mathrm{k}} * / \mathrm{P}_{\mathrm{j}} / /$ components of the $\mathrm{k}^{\text {th }}$ image

Step 3: read $i$; */ read component $i$, from database Ds
Step 4: $\mathrm{Cen}_{\mathrm{i}} ; *$ / $\mathrm{Cen}_{\mathrm{i}}$

Step 5: find $\mathrm{X}_{\mathrm{i}} ; * / \mathrm{X}_{\mathrm{i}}$

Step 6: calculate $\mathrm{Fea}_{\mathrm{i} 1}, \mathrm{Fea}_{\mathrm{i} 2} \ldots \mathrm{Fea}_{\mathrm{i} 8}$

end;

end;

\section{CBIR Algorithm}

Begin

I $\leftarrow$ Scan Image

$\mathrm{J} \leftarrow$ Filter Image.

Step 1: assign output Pixel Value[width][ height]

Step 2: assignwindow[win width * win height]

Step 3:image_edgex := (win width / 2 ) rounded

Step 4:image_edgey $:=($ win height $/ 2$ ) rounded

Step 5: for $\mathrm{x}$ from image_edgex to image width image_edgex

for y from image_edgey to image height -image_edgey $\mathrm{i}=0$

for fx from 0 to win width

for fy from 0 to win height

win[i] := inputPixelValue[x $+\mathrm{fx}-$

image_edgex $][y+$ fy - image_edgey]

$\mathrm{i}:=\mathrm{i}+1$

sort entries in win[]

outputPixel[x][y] := win[win width * win height / 2]

Step 6:similarity_measure $\leftarrow$ Euclidian Distance

Step 7: Object $\leftarrow$ CBIR (I)

\section{RESULT AND DISCUSSION}

In this section the results of the research are presented. A comparative study for existing [8] and proposed approach is shown and proved that a proposed approach is better as compared to existing one in terms of the parameters defined below: matching time, precision, recall, accuracy and efficiency. For result comparison recall and precision are used that are defined as below:

Recall: It may be defined as relevant images that are extracted divided by total number of relevant images.

Precision: It may be defined as number of relevant images searched divided by total images retrieved by that search.

The experimental results are given in the Table 2 to Table 7. For better presentation the experimental results are also represented in a plotted form which are shown in figure 3 to figure7.

Table II: Comparison of time taken and number matching points calculated by the existing [8] and proposed technique.

\begin{tabular}{|c|c|c|c|c|c|}
\hline \multicolumn{2}{|c|}{$\begin{array}{c}\text { Key Points in } \\
\text { Image }\end{array}$} & \multicolumn{2}{c|}{$\begin{array}{c}\text { Existing } \\
\text { approach matching }\end{array}$} & \multicolumn{2}{c|}{$\begin{array}{c}\text { Proposed } \\
\text { Approach Matching }\end{array}$} \\
\hline $\begin{array}{c}\text { Que } \\
\text { ry } \\
\text { Ima } \\
\text { ge }\end{array}$ & $\begin{array}{l}\text { Dataset } \\
\text { images }\end{array}$ & $\begin{array}{c}\text { Time of } \\
\text { Matching } \\
\text { (Sec) }\end{array}$ & $\begin{array}{c}\text { Matchi } \\
\text { ng } \\
\text { Points } \\
\text { inside } \\
\text { images }\end{array}$ & $\begin{array}{c}\text { Time of } \\
\text { Matching } \\
\text { (Sec) }\end{array}$ & $\begin{array}{c}\text { Match } \\
\text { ing } \\
\text { points } \\
\text { inside } \\
\text { image } \\
\text { sec }\end{array}$ \\
\hline 227 & 289 & 0.045 & 136 & 0.023 & 143 \\
\hline 747 & 640 & 0.250 & 347 & 0.221 & 365 \\
\hline
\end{tabular}

Published By:

Blue Eyes Intelligence Engineering

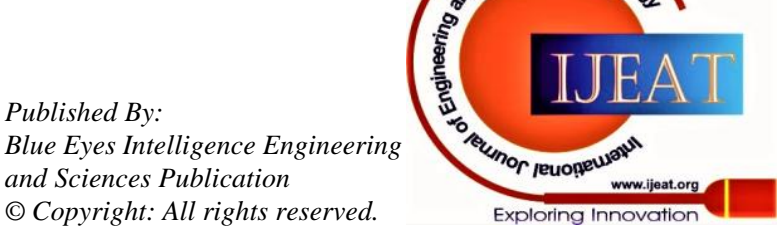


Improved Content-Based Image Retrieval Technique for Query Generation in Mobile Networks

\begin{tabular}{|c|c|c|c|c|c|}
\hline $\begin{array}{c}311 \\
4\end{array}$ & 2633 & 4.970 & 673 & 3.865 & 767 \\
\hline $\begin{array}{c}667 \\
1\end{array}$ & 6776 & $\begin{array}{c}48.79 \\
0\end{array}$ & 896 & 42.412 & 971 \\
\hline
\end{tabular}

Fig. 4. Accuracy vs No of images curves for the Existing [8] and Proposed Approach.

Fig 4 defines the Accuracy curve for existing and proposed technique. It is clearly observed that existing technique is not

Table III: Comparative Analysis of Recall vs Precision in existing [8] and proposed approach

\begin{tabular}{|l|l|l|}
\hline $\begin{array}{c}\text { Technique } \\
\text { No of tmages }\end{array}$ & Existing & Proposed \\
\hline 0 & 0 & 0 \\
\hline 1 & 0.35 & 0.4 \\
\hline 2 & 0.4 & 0.45 \\
\hline 3 & 0.41 & 0.5 \\
\hline 4 & 0.48 & 0.55 \\
\hline 5 & 0.54 & 0.59 \\
\hline 6 & 0.6 & 0.63 \\
\hline 7 & 0.65 & 0.68 \\
\hline 8 & 0.73 & 0.78 \\
\hline
\end{tabular}

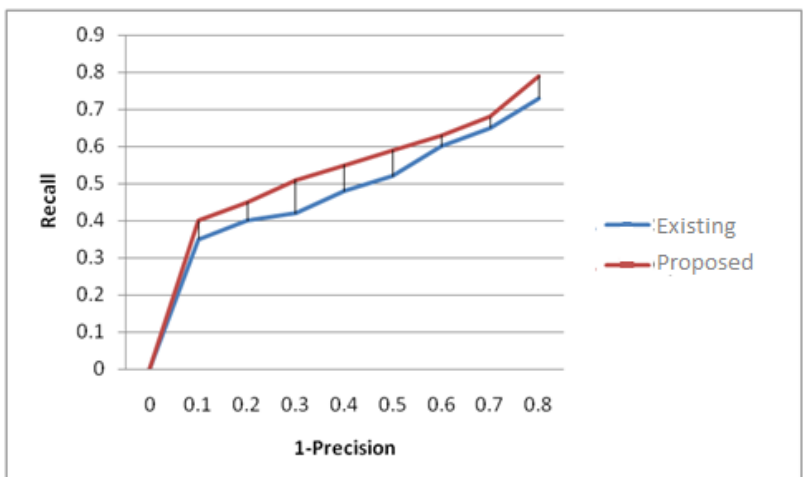

Fig. 3. Recall versus 1-Precision plots for the Existing [8] and proposed approach.

Fig 3 represents Recall vs Precision curve for existing and proposed technique which proves that the proposed technique outperforms the existing technique in terms of precision and recall values.

Table IV: Comparative Analysis of Accuracy in existing [8] and proposed approach

\begin{tabular}{|l|l|l|}
\hline $\begin{array}{c}\text { Technique } \\
\text { No of Images }\end{array}$ & Existing & Proposed \\
\hline 20 & 98.2 & 99 \\
\hline 40 & 98.1 & 98.6 \\
\hline 60 & 97.6 & 98 \\
\hline 80 & 97.5 & 98.4 \\
\hline 100 & 97.4 & 98.2 \\
\hline
\end{tabular}

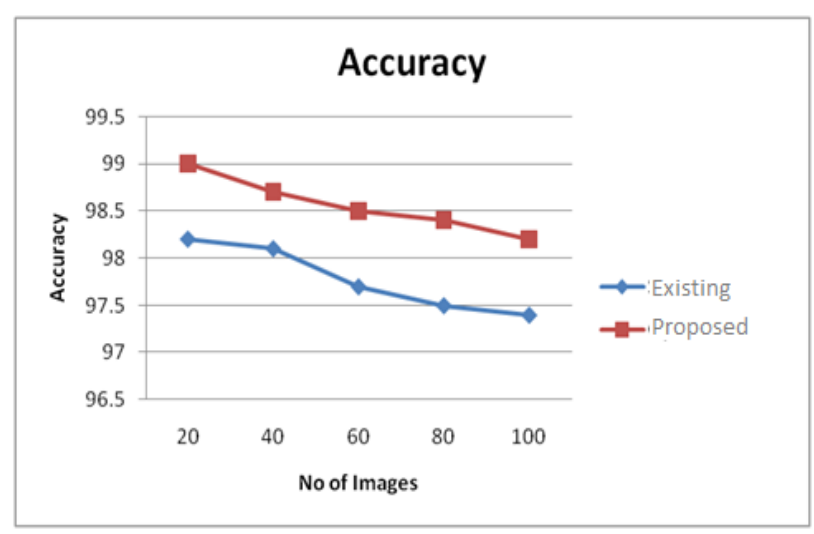

Retrieval Number: F1626089620/2020@BEIESP as accurate as proposed technique. Value of proposed technique in this graph is about $99 \%$ whereas for existing is $98.2 \%$.

Table V: Comparative Analysis of Efficiency in existing and proposed approach

\begin{tabular}{|l|l|l|}
\hline $\begin{array}{l}\text { Fechnique } \\
\text { No of Images }\end{array}$ & Existing & Proposed \\
\hline 20 & 98.6 & 99.4 \\
\hline 40 & 98.5 & 99.3 \\
\hline 60 & 98.4 & 99.2 \\
\hline 80 & 98.2 & 99.1 \\
\hline 100 & 98 & 99 \\
\hline
\end{tabular}

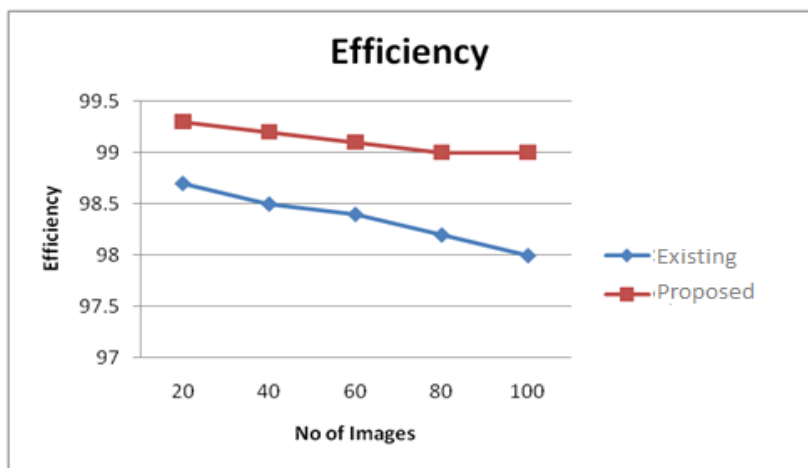

Fig. 5. Efficiency vs No of images values for the original and optimized proposed approach matching methods

Fig 5 defines the efficiency curve for existing and proposed technique. It is clear that existing technique is not as efficient as proposed technique with values $98.7 \%$ and $99.2 \%$.

Table VI: Comparative Analysis of Recall in existing [8]and proposed approach

\begin{tabular}{|l|l|l|}
\hline $\begin{array}{c}\text { Fechnique } \\
\text { No of Images }\end{array}$ & Existing & Proposed \\
\hline 20 & 98 & 99 \\
\hline 40 & 97.8 & 98.8 \\
\hline 60 & 97.4 & 98.6 \\
\hline 80 & 97.1 & 98.3 \\
\hline 100 & 97 & 98 \\
\hline
\end{tabular}

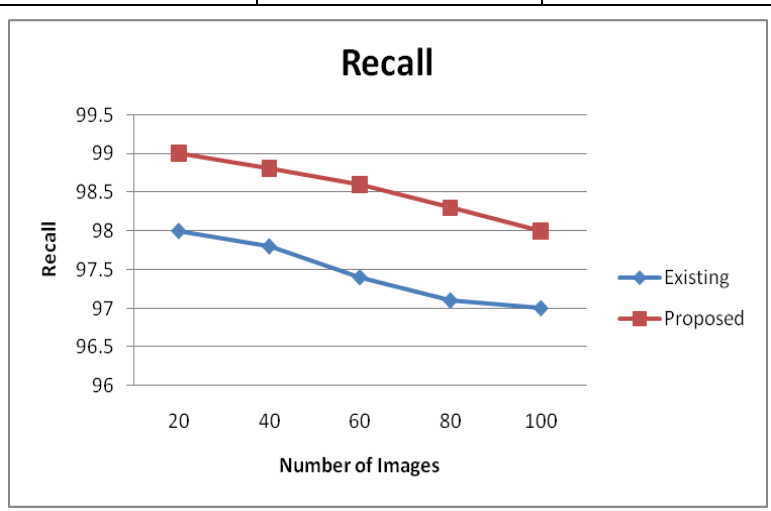

Fig. 6. Recall vs No of images curves for the original and optimized proposed approach matching methods

Blue Eyes Intelligence Engineering and Sciences Publication

(c) Copyright: All rights reserved.

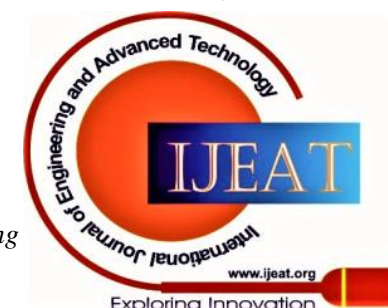


Fig 6 defines the Recall curve for existing and proposed technique. It is clearly seen that proposed technique is much better than existing technique.

A value of proposed and existing technique in this graph is about $99 \%$ and $98 \%$.

Table VII: Comparative Analysis of Precision in existing [8] and proposed approach

\begin{tabular}{|l|l|l|}
\hline $\begin{array}{c}\text { Technique } \\
\text { No of Images }\end{array}$ & Existing & Proposed \\
\hline 20 & 98.1 & 99.4 \\
\hline 40 & 98 & 99.2 \\
\hline 60 & 97.5 & 98.8 \\
\hline 80 & 97.2 & 98.4 \\
\hline 100 & 97.1 & 98.2 \\
\hline
\end{tabular}

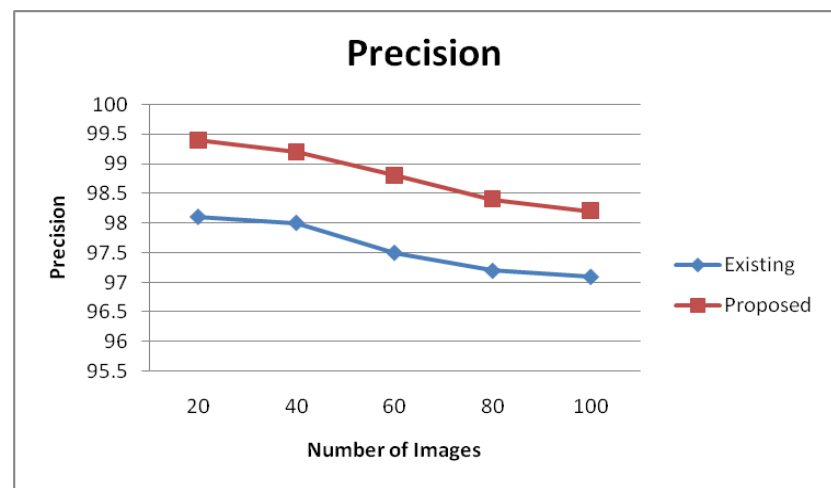

Fig. 7. Precision vs No of images curves for the original and optimized proposed approach matching methods

Fig 7 defines the Precision curve for both existing and proposed technique. It is clear that existing technique is not as effective as proposed technique values being $99.5 \%$ and 98.2 for proposed and existing respectively.

\section{CONCLUSION}

CBIR at the moment continues to be much an enquiry topic. The technology is thrilling however undeveloped and few operational image archives have however shown serious interest in approval. The appliance areas that are likely to profit from the acquisition of CBIR are those where level one technique is directly used.

In this work we have enforced many CBIR techniques that extract GLCM based features and then based upon VGG-16 architecture results are generated. So, in the proposed work RCNN based technique is used on VGG-16 architecture and query image is send to cloud by mobile user and in the end, results are generated. Within the analysis of work, it is observed that the projected technique is generating quite improved results as compared to the existing [8] techniques. The result part describes that the proposed approach is performing better than existing approach in terms of different parameters such as accuracy, efficiency, matching time, precision and recall curves.

In future, one can work on how to generate same results by taking the noisy images into consideration.

\section{REFERENCES}

1. Boparai, N.K.; Chhabra, A.; "A Hybrid Approach for Improving Content Based Image Retrieval Systems", International Conference on Next Generation Computing Technologies, 2015, pp: 944-949
2. Bose, S.; Pal, A.; Mallick, J.; Kumar, S.; Rudra, P.; "A Hybrid Approach for Improved Content-based Image Retrieval using Segmentation", 2015, pp: 2-21

3. Mistry, Y.; Ingole, D.T.; Ingole, M.D.; "Content based image retrieval using hybrid features and various distance metric", Journal of Electrical Systems and Information Technology, 2016, pp: 1-15

4. Zhang, C.; Li, J.; Wang, S.; Wang, Z.; "An encrypted medical image retrieval algorithm based on DWT-DCT frequency domain", IEEE, 2017, pp: 7-9

5. Raza, A.; Dawood, H.; Dawood, H.; Shabbir, S.; Mehboob, R.; Banjar, A.; "Correlated Primary Visual Texton Histogram Features for Content Base Image Retrieval”, IEEE, 2018, pp: 46595-46616

6. Swati, Z.N.K.; Zhao, Q.; Kabir, M.; Ali, F.; Zakir, A.; Ahmad, S.; Lu, J.; "Content-Based Brain Tumor Retrieval for MR Images Using Transfer Learning”, IEEE, ISSN: 2169-3536, 2019, pp: 1-13

7. Cai, Y.; Li, Y.; Qiu, C.; Ma, J.; Gao, X.; "Medical Image Retrieval Based on Convolutional Neural Network and Supervised Hashing", IEEE, Vol: 7, 2019, pp: 51877-51885

8. Liu, F.; Wang, Y.; Wang, F.; Zhang, Y.; Lin, J.; "Intelligent and Secure Content-Based Image Retrieval for Mobile Users”, IEEE, Vol: 7, 2019, pp: 119209-119222

9. K. K. Kumar, "CBIR: Content Based Image Retrieval,” In National Conference on Recent Trends in Information/ Network Security, vol. 23, 2010.

10. Y. Rui, T. Huang, M. Ortega, and S. Mehrotra. Relevance feedback: a power tool for interactive content-based image retrieval. IEEE Transactions on Circuits and Video Technology, 1998.

11. Y. Wu and A.Zhang. A feature reweighting approach for relevance feedback in image retrieval. In proceedings of International Conference on Image Processing (ICIP 2002), Rochester, new York, September 2002.

12. H.Muller, A. Rosset, J.-P. Vallee, and A. Geissbuhler, "Comparing features sets for content-based image retrieval in a medical case database," Proc.SPIE, vol. 5371, 99-110,Apr.2004.

13. Rajput, M.; "Content Based Image Retrieval Using Color and Texture Feature with Distance Matrices", International Journal of Scientific and Research Publications, Vol: 7, Issue 8, August2017, pp: 512-523.

14. Jesi, V.E.; Govindarajan, S.; Jayanthi, M.; "A State of Art on Content Based Image Retrieval systems", International Journal of Recent Technology and Engineering, Vol: 8 Issue-2S4, July 2019, pp: 297-310.

15. Lande, M.V.; Bhanodiya, P.; Jain, P.; "Efficient Content Based Image Retrieval Using Color and Texture", International Journal of Scientific \& Engineering Research, Vol: 4, Issue 6, June-2013, pp: 121-131.

\section{AUTHORS PROFILE}

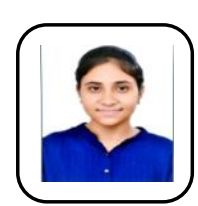

Barinder Kaur received the B.Tech degree in computer science and engineering from GNE college Ludhiana, in 2018 and currently pursuing masters in computer science and engineering from Punjabi University, Patiala. Her research interest includes Digital Image Processing.

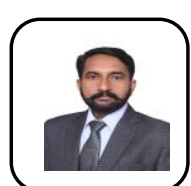

Madan Lal has received his B.Tech degree in Computer Science and Engineering from Guru Nanak Dev University, Amritsar in 1998 and M.tech in Computer Engineering from Punjabi University, Patiala, in 2007. He obtained his PHd. Degree from Punjabi University Patiala, in 2018. He has published more than 30 papers in referred International Journals and Conference Proceedings. His research interest includes Computer Graphics and Digital Image Processing.

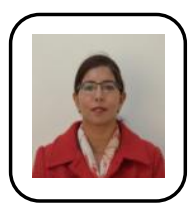

Jagroop Kaur is working as Assistant professor in department of computer engineering at Punjabi University Patiala. She obtained her master degree in computer Science and Engineering from Punjabi University Patiala in 2007. She has more than 18 years of teaching experience. Her area of interest is Natural Language Processing, Machine Learning and Social Media Text Mining. Jagroop Kaur is the corresponding author and can be contact at jagroop_80@rediffmail.com

Blue Eyes Intelligence Engineering

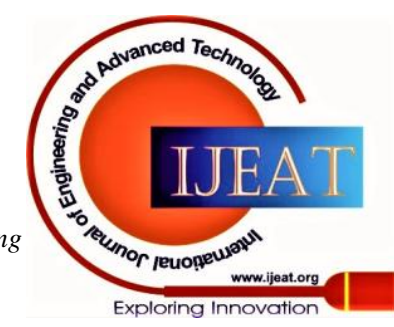

